\title{
Frühes Ansprechen sagt späteren Erfolg voraus
}

Fragestellung: Wann soll die antipsychotische Behandlung eines Patienten mit schizophrener Störung bei fehlender Besserung umgestellt werden?

Hintergrund: Viele Studien untersuchten diese Fragestellung, verwendeten aber unterschiedliche Definitionen für das frühe Therapieansprechen $(<20 \%$ bis $<50 \%$ des Ausgangswerts) und den späteren Therapieerfolg sowie unterschiedliche Zeiträume (zwei bis sechs Wochen für das frühe Therapieansprechen). Daher sind die Therapieleitlinien in dieser Hinsicht uneinheitlich und wenig evidenzbasiert. Diese Metaanalyse ging der Frage nach, ob ein fehlendes Therapieansprechen nach zwei Wochen eine fehlende spätere Response mit hoher Sensitivität vorhersagen kann.

Patienten und Methodik: In die Untersuchung wurden 34 Studien (insgesamt 9.460 Patienten) eingeschlossen, davon 29 randomisierte klinische Studien

Samara MT, Leucht C, Leeflang MM et al. Early improvement as a predictor of later response to antipsychotics in schizophrenia: A diagnostic test review. Am J Psychiatry 2015; 172: 617-29 (RCT). Die Datensätze von 32 Studien wurde von den Autoren zur Verfügung gestellt und flossen in die Auswertung ein. Bei sechs Studien wurde Patienten mit Ersterkrankungen untersucht.
Eingeschlossen wurden Studien, welche die frühe Therapieantwort unter Antipsychotika nach zwei Wochen anhand des PANSS (Positive and Negative Syndrom Scale) oder des BPRS (Brief Psychiatric Rating Scale) erhoben und zudem den späteren Therapieerfolg nach vier bis zwölf Wochen gemessen hatten. Als primärer Outcome-Parameter wurde die Vorhersage des späteren Therapieversagens am Studienende $(<50 \%$ des PANSS oder BPRS) anhand des ausbleibenden frühen Therapieansprechens nach zwei Wochen (<20\% des PANSS oder BPRS) gewählt.

Ergebnisse: Ein Ausbleiben eines frühen Therapieansprechens konnte einen fehlenden Therapieerfolg mit einer Spezifität von $86 \%$ und einem positiven prädiktiven Wert von $90 \%$ vorhersagen. Ein höherer Schweregrad der Ausgangssymptome oder ein kürzerer Verlauf der Erkrankung gingen mit einer höheren Spezifität einher. Keine signifikanten Effekte ergaben sich für das Geschlecht, das Studiendesign und die Substanzklassen der Antipsychotika (Antipsychiotika erster und zweiter Generation).

Schlussfolgerungen: Bei schizophrenen Patienten mit fehlender Verbesserung der Symptomatik nach zwei Wochen antipsychotischer Behandlung ist eine spätere relevante Besserung meist nicht zu erwarten, diese Patienten werden in der Regel von einer Umstellung der Medikation profitieren.

\section{- Kommentar von Andreas Konrad, Andernach}

\section{Bei fehlendem Ansprechen Therapie nach 14 Tagen umstellen}

Wie lange soll eine antipsychotische Behandlung bei fehlendem Therapieansprechen fortgeführt werden? Dies ist eine klinisch häufige und bedeutsame Frage. In den Leitlinien gibt es hierzu verschiedene Angaben zwischen zwei und sechs Wochen, die jedoch auf geringer Evidenz basieren.

Die vorliegende Metaanalyse geht auf diese Frage ein und analysiert hierzu die Datensätze von insgesamt 34 ausgewählten Studien, größtenteils RCT. Die Auswahl der Zielkriterien ist schlüssig und klinisch sinnvoll, so entspricht eine Verbesserung von $20 \%$ im PANSS oder BPRS einer "minimalen Verbesserung" im CGI (Clinical Global Impressions Scale), während eine Verbesserung von $50 \%$ im PANSS oder BPRS einer "deutliche Verbesserung" im CGI entspricht. Einschränkungen der Studie hinsichtlich der Übertragbarkeit auf den klinischen Alltag ergeben sich in folgender Hinsicht: Aufgrund der Analyse von vorwiegend RCT ist davon auszugehen, dass enge Einschlusskriterien bei der Auswahl der Patienten bestanden, insbesondere hinsichtlich Komorbiditäten. Wie auch in anderen Studien im Bereich der schizophrenen Erkrankungen bestand zudem eine hohe Dropout-Rate. In den meisten Studien war eine Komedikation zugelassen, diese könnte jedoch auch die Ergebnisse beeinflussen, etwa eine initiale Benzodiazepinbehandlung.

Eine Analyse von Einzelsubstanzen konnte aufgrund der Fallzahlen nur für Haloperidol, Risperidon, Olanzapin und Ami- sulprid durchgeführt werden. Hierbei ergaben sich ebenso wie bei der Analyse der beiden Substanzgruppen (Typika und Atypika) keine signifikanten Unterschiede. Die genannten Substanzen stellen jedoch aufgrund der Unterschiede inbesondere hinsichtlich der Rezeptorprofile eine gute Auswahl dar.

Es ist darauf zu achten, dass Therapieversuche immer mit einer ausreichend hohen Dosis durchgeführt werden. Durch ein möglichst frühzeitiges Umstellen einer wirkungslosen Behandlung können den Patienten längere wirkungslose und unter Umständen nebenwirkungsbelastete Therapieversuche erspart bleiben. Für die Frage, ob dann eine Umstellung der Medikation oder eine Augmentationsstrategie vielversprechender ist, gibt es bis dato allerdings wenig Evidenz.

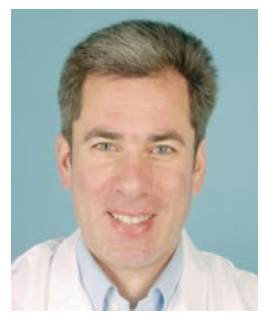

PD Dr. med. Andreas Konrad, Andernach

Chefarzt Allgemeinpsychiatrie II Rhein-Mosel-Fachklinik Andernach E-Mail: a.konrad@rmf.landeskrankenhaus.de 